\title{
Introduction to the Special Section on the 2018 USENIX Annual Technical Conference (ATC'18)
}

This special section of the ACM Transactions on Storage presents some of the highlights of the 2018 USENIX Annual Technical Conference (ATC'18). Over the years, USENIX ATC has evolved into a community of researchers and practitioners working on a diverse and expanding set of research topics; the conference represents some of the latest and best work being done, and this year was no different. ATC'18 received a record number of 377 submissions. Of these, we selected three high-quality storage-related articles for publication in this special section of ACM Transactions on Storage.

The first article, which was also selected as one of the best papers in ATC'18, is “TxFS: Leveraging File-System Crash Consistency to Provide ACID Transactions" by Yige Hu, Zhiting Zhu, Ian Neal, Youngjin Kwon, Tianyu Cheng, Vijay Chidambaram, and Emmett Witchel. Building transactional systems is complex and error-prone. The authors of this article introduce a novel approach to build a transactional file system by taking advantage of the mature, well-tested filesystem journal feature. Compared to earlier transactional file systems, it is easy to develop and use. It also demonstrates performance boosts for a number of different workloads.

The second article is "CGraph: A Distributed Storage and Processing System for Concurrent Iterative Graph Analysis Jobs” by Yu Zhang, Jin Zhao, Xiaofei Liao, Hai Jin, Lin Gu, Haikun Liu, Bingsheng He, and Ligang He. Nowadays, distributed graph processing platform, which handles massive Concurrent iterative Graph Processing (CGP) jobs, is widely used. However, existing distributed systems face a high ratio of data access cost to computation for the CGP jobs, which incurs low throughput. The authors observed that this phenomenon happened because these CGP jobs need to repeatedly traverse the shared graph structure. They then propose exploiting the observed spatial and temporal correlations between the data accesses of these jobs to enable multiple concurrent iterative graph processing jobs. Hence, it efficiently shares the graph data and provides higher throughput.

The final article is "SolarDB: Towards a Shared-Everything Database on Distributed Log-Structured Storage” by Tao Zhu, Zhuoyue Zhao, Feifei Li, Weining Qian, Aoying Zhou, Dong Xie, Ryan Stutsman, Haining Li, and Huiqi Hu. Supporting transactions in distributed database with outstanding performance has been a complex issue to tackle. In this work, the authors describe how to build a shared-everything distributed relational database that achieves dramatically faster, fine-grained, and non-blocking transactions, based on log-structured trees and new control mechanisms.

Haryadi Gunawi and Benjamin Reed Program Committee Co-Chairs

(C) 2019 Copyright held by the owner/author(s). 1553-3077/2019/05-ART8

https://doi.org/10.1145/3322100 